\title{
Exploring a Simple Stochastic Mathematical Model Including Fear with a Linear Functional Response
}

\author{
Aytül Gökçe \\ Department of Mathematics, Faculty of Arts and Sciences, Ordu University, Ordu, Turkey
}

\author{
Article Info \\ Keywords: Linear functional response, \\ Prey-predator interactions, Stability \\ analysis, White Gaussian noise \\ 2010 AMS: 37A50, 9210 \\ Received: 11 August 2021 \\ Accepted: 4 November 2021 \\ Available online: 1 December 2021
}

\begin{abstract}
This paper concentrates on a simple population model incorporating fear. Firstly, positivity and steady state analysis are performed, where the theoretical investigations show that change in the level of fear in prey population does not effect the local stability of the system around each equilibria (either stable or unstable). For the deterministic model, the numerical simulations are plotted for the density of prey species as a function of various system parameters. The stability analysis of the coexisting state shows that only transcritical bifurcation, where the steady states intersect, is observed. Secondly, the model is analysed with Gaussian noise term incorporated in the prey's death rate. The model comprising noise term turns the system into stochastic differential equations and irregular noise related oscillations are observed in the densities of both species.
\end{abstract}

\section{Introduction}

Due to the complexity of the ecosystem, it is often challenging to predict the effects of the various parameters in the dynamics of species using only statistical information. Therefore it is very crucial to incorporate mathematical formulations into research to better comprehend the reasons and outcomes of the dynamics of species in the ecosystem. In particular, theoretical formulations may help to foresee the required conditions for controlling ecological balance. In this context, the latest theories regarding the evolution of predator-prey type interactions shed lights on the prediction of population dynamics for multiple species, including their response to environmental conditions.

In mathematical terms, the impact of prey species response to predator species has been analysed using a wide variety functional responses. Here, a functional response refers to the consumption rate of a predator on prey [1]. The most common functional responses that have been used to model predator and prey interactions are Holling type I (i), Holling type II (ii) and Holling type III (iii) functional responses, classified by Crawford Stanley Holling [2]-[4]. In this paper, Holling type I, leading to a linear functional response will be taken into consideration.

The presence of prey species directly or indirectly depends on the presence of predators. Most of the available models analyse the direct influence of predator species on the prey species such as predation via direct killing. However, the change in the prey density may have also been affected due to indirect factors including psychological conditions such as fear. In fact, scared prey species may change their habitat and live in various conditions, where the quality of the new habitat may lead an increase or decrease in the density of prey population. There are many experimental studies to explore the role of fear in a population [5]-[7]. From the mathematical point of view, the fear effect has been thoroughly explored in recent years. For example, the role of fear in a prey-predator system with BeddingtonDeAngelis functional response has been studied by Pal et al. [8]. The instability of a population dynamics due to large fear parameters has been analysed by Wang and Zou 
[9]. The impact of group defence along with fear has been discussed by Sasmal and Takeuchi [10]. See [11]-[13] for other comprehensive works taking the fear effect into account.

Although previously studied deterministic models do not take stochastic effects into account, the random fluctuations may occur in nature due to climate change or some short term diseases which may affect the evolution of species. In fact these fluctuations may appear in any biological process. Therefore, it is worth to incorporate environmental noise in the model to better capture the dynamics of species in ecology. Many scientist have studied models with Gaussian noise from various point of view, including stochastic models with stage structure [14], foraging [15], anti-predator defence [13], intra-specific competition [16], leading to stochastic differential equations.

This paper is organised as follows: The deterministic model given in [17] is revisited in Section 2, where positivity of the model, its steady states and local stability analysis are derived respectively in Sections 2.1, 2.2 and 2.3. In Section 3, incorporation of the white Gaussian noise in the prey's death rate is discussed. Numerical simulations resulting from local stability analysis, as well as the comparison between deterministic model and stochastic model are demonstrated in Section 5 . Furthermore the conditions under which prey and predator species go to extinction are provided in Section 4.

\section{Deterministic dynamics of the model}

A deterministic model based on the paper [17] is written as in the following:

$$
\frac{\mathrm{d} x}{\mathrm{~d} t}=r_{0} x f_{1}(k, y)-d x-a x^{2}-f_{2}(x) y:=\mathscr{A}_{1}(x, y, t), \quad \frac{\mathrm{d} y}{\mathrm{~d} t}=y\left(-m+c f_{2}(x)\right):=\mathscr{B}_{1}(x, y, t),
$$

where parameters $r_{0}, k, d, a$ and $m$ respectively stand for the birth rate for prey, the level of fear due to predator, the natural death rate of prey, the death rate of prey as a result of intra-species competition and the natural death rate of predator. Here $c$ denotes the conversion rate from prey to predator biomass. All parameters stated in the model are positive for their biological meaning. The function $f_{1}$ denotes the fear factor and function $f_{2}$ represents linear functional response.

The function $f_{1}(k, y)$ satisfies the following conditions:

$$
\begin{aligned}
& f_{1}(0, y)=1, \quad f_{1}(k, 0)=1, \\
& \lim _{k \rightarrow \infty} f_{1}(k, y)=0, \quad \lim _{y \rightarrow \infty} f_{1}(k, y)=0, \\
& \frac{\partial f_{1}(k, y)}{\partial k}<0, \quad \frac{\partial f_{1}(k, y)}{\partial y}<0 .
\end{aligned}
$$

and $f_{2}(x)=p x$ is taken as a linear function. To make the system mathematically attainable, fear factor is adopted in a form of $f_{1}(k, y)=1 / 1+k y$, that satisfies the conditions given in equation (2.2) [17].

\subsection{Positivity}

It is straightforward to show that all solutions $(x(t), y(t))$ of the model given by (2.1) are non-negative with positive initial conditions, i.e. $\left(x_{0}, y_{0}\right) \in \mathbb{R}_{+}^{2}$ for $\forall t \in \mathbb{R}_{+}$. Using the first equation in model (2.1), one can write that

$$
\frac{\mathrm{d} x}{x}=\mathscr{A}_{2}(x, y, t) \mathrm{d} t
$$

where $\mathscr{A}_{2}(x, y, t)=r_{0} /(1+k y)-d-a x-p y$. Integrating both sides of equation (2.3) it follows that

$$
\ln x-\ln x_{0}=\int_{0}^{t} \mathscr{A}_{2}\left(x, y, s^{\prime}\right) \mathrm{d} s^{\prime}
$$

Subtracting $x$ from equation (2.4) leads to

$$
x(t)=x_{0} \exp \left\{\int_{0}^{t} \mathscr{A}_{2}\left(x, y, s^{\prime}\right) \mathrm{d} s^{\prime}\right\}, \quad \forall t>0 .
$$

Similarly, positivity of the predator variable $y$ can be shown as

$$
\frac{\mathrm{d} y}{y}=\mathscr{B}_{2}(x, t) \mathrm{d} t, \Rightarrow y=y_{0} \exp \left\{\int_{0}^{t} \mathscr{B}_{2}\left(x, s^{\prime}\right) \mathrm{d} s^{\prime}\right\}, \quad \forall t>0 .
$$

where $\mathscr{B}_{2}(x, s)=-m+c p x$. Since $\left(x_{0}, y_{0}\right) \in \mathbb{R}_{+}^{2}$ for $\forall t>0$, it is obtained that $x(t)>0$ and $y(t)>0$. Thus the interior of $\mathbb{R}_{+}^{2}$ is an invariant set of model (2.1). This is also biologically meaningful as the densities of prey and predator species are expected to be non-negative. 


\subsection{Steady state analysis}

The model given by (2.1) has three steady states which can be found using $\mathscr{A}_{1}(x, y, t)=0$ and $\mathscr{B}_{1}(x, y, t)=0$. The first steady state is the trivial equilibrium $E_{0}=(0,0)$ where both prey and predator populations go to extinction. The second steady state is semi trivial state $S_{1}=\left(\left(r_{0}-d\right) / a, 0\right)$ bywhich prey population still exists in the system in the absence of predator. The last steady state is coexisting state, say $S_{2}=(x *, y *)$, where both prey and predator populations exist in the system. Here $x *=m / c p$ can be easily found using $\mathscr{B}_{1}(x, y, t)=0$ for $y \neq 0$. The critical value for predator $y *$ are obtained solving

$$
\frac{r_{0}}{1+k y}-d-a x-p y=0
$$

which can be rewritten for coexisting state as

$$
(p y *+h)(k y+1)=r_{0}, \quad h=d+a m / c p .
$$

Here equation (2.5) has two roots one of which may be positive (thus biologically meaningful) under the condition $r_{0}>h$, for which coexisting state $S_{2}=(x *, y *)$ exists.

In addition the nullclines of the model can be similarly found:

$$
\begin{gathered}
x=m / c p, \quad(\text { straight line for predator nullcline }) \\
p k y^{2}+(h k+p) y+h-r_{0}=0, \quad(\text { parabolic curve for prey nullcline) } .
\end{gathered}
$$

\subsection{Local stability analysis around coexisting state}

Stability of the system (2.1) can be determined using linearisation argument, where the prey and predator densities are perturbed from their steady state. Considering

$$
x=x *+\tilde{x}, \text { and } y=y *+\tilde{y},
$$

where accents $\sim$ represent the perturbed variables. Substituding these in the original model the following linearised system of equations are obtained:

$$
\begin{aligned}
\frac{\mathrm{d} \tilde{x}}{\mathrm{~d} t} & =\left(\frac{r_{0}}{1+k y *}-d-2 a x *-p y *\right) \tilde{x}+\left(-\frac{k r_{0} x *}{(1+k y *)^{2}}-p x *\right) \tilde{y}, \\
\frac{\mathrm{d} \tilde{y}}{\mathrm{~d} t} & =c p y * \tilde{x}+(c p x *-m) \tilde{y} .
\end{aligned}
$$

The coefficient matrix for the above linear system of equations can be written as

$$
\mathscr{M}_{J}=\left(\begin{array}{cc}
\frac{r_{0}}{1+k y *}-d-2 a x *-p y * & -\frac{k r_{0} x *}{(1+k y *)^{2}}-p x * \\
c p y * & c p x *-m
\end{array}\right)
$$

for which corresponding characteristic polynomial are obtained using $\operatorname{Det}\left(\mathscr{M}_{J}-\mu \mathscr{I}\right)=0$ where $\mathscr{I}$ is a $2 \times 2$ unit matrix and $\mu$ is the eigenvalues of the system for local stability. The characteristic equation can be explicitly written as

$$
\mu^{2}-\mathscr{E}_{1}\left(\mathscr{M}_{J}\right) \mu+\mathscr{E}_{2}\left(\mathscr{M}_{J}\right)=0,
$$

where

$$
\begin{aligned}
& \mathscr{E}_{1}=\frac{r_{0}}{1+k y *}-d-2 a x *-p y *+c p x *-m, \\
& \mathscr{E}_{2}=\left(\frac{r_{0}}{1+k y *}-d-2 a x *-p y *\right)(c p x *-m)+\left(\frac{k r_{0} x *}{(1+k y *)^{2}}+p x *\right) c p y *
\end{aligned}
$$

and the roots for the equation (2.6) is given as

$$
\mu_{1,2}=\frac{1}{2}\left[\mathscr{E}_{1}\left(\mathscr{M}_{J}\right) \pm \sqrt{\mathscr{E}_{1}\left(\mathscr{M}_{J}\right)-4 \mathscr{E}_{2}\left(\mathscr{M}_{J}\right)}\right]
$$

It is worth noting that $\mathscr{E}_{1}$ and $\mathscr{E}_{2}$ respectively stand for trace and determinant of the matrix $\mathscr{M}_{J}$.

In Figure 2.1, stability of the prey density is shown with regard to parameters $p, k, a, r_{0}$ that respectively stand for the strength of linear functional response, rate of fear due to predator, the death rate of prey due to intraspecies competition and the birth rate of prey. As seen there is a switch in the stability when eigenvalues crosses the transcritical bifurcation, that is a typical case in population models referring to invasion. In fact transcritical bifurcation demonstrates the onset of coexisting state in the system $[18,19]$. Here the dashed line indicates unstable state where the number of eigenvalues with positive real part is 1 , whereas the straight line indicates the stable state with both eigenvalues are found with negative real part. 
(a)

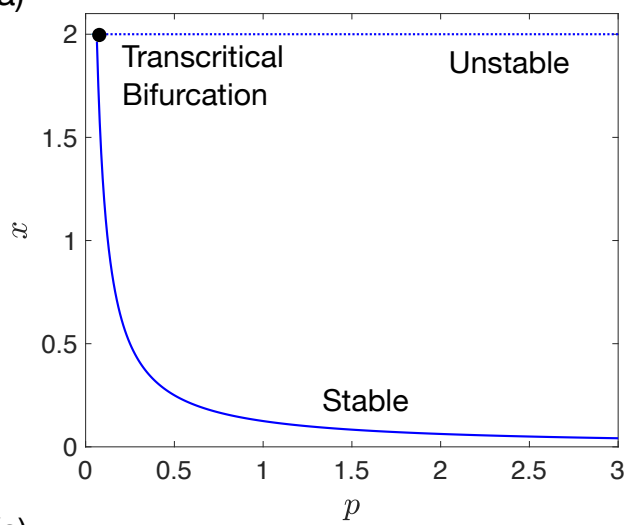

(c)

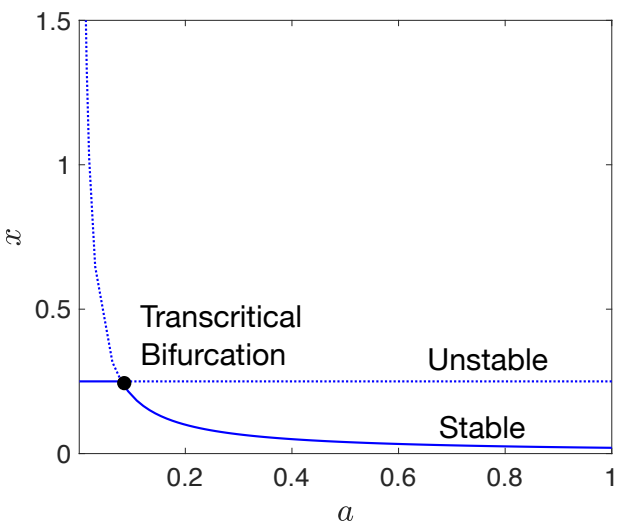

(b)

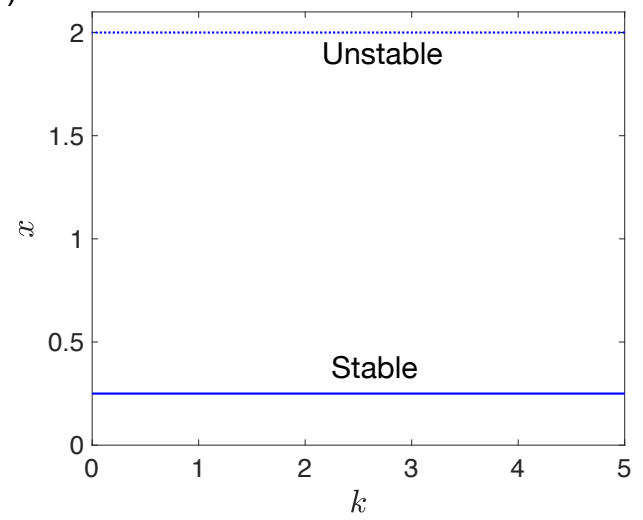

(d)

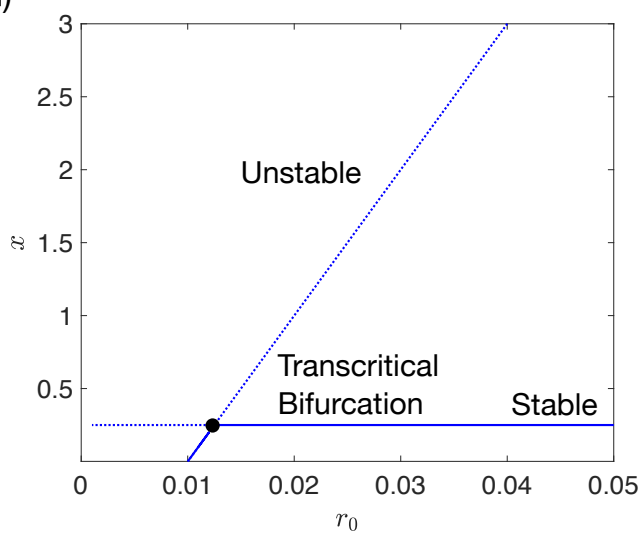

Figure 2.1: Transcritical bifurcation occurs in the model (2.1) with respect to various parameters, i.e. $p, k, a$ and $r_{0}$. See text around the system (2.1) for biological interpretations of these parameters.

\section{Analysis of the model with white Gaussian noise}

Deterministic model provided in Section 2 does not comprise the role of environmental fluctuations which may be any unpredictable factor including the quality of food, climate change, diseases as well as temperature [14, 20]. Hence incorporating random noise in model parameters may significantly alter dynamics of both prey and predator species. Although any parameter of the model may be affected by environmental noise, the uncertain growth and death rates may be particularly influenced, see for example [16, 21, 22].

$$
\mathrm{d} x=\left[r_{0} x f_{1}(k, y)-d x-a x^{2}-p x y\right] \mathrm{d} t-\varepsilon x \mathrm{~d} \beta, \quad \mathrm{d} y=[c p x y-m y] \mathrm{d} t .
$$

which can be also written as

$$
\mathrm{d} x=\mathscr{A}_{1}(x, y, t) \mathrm{d} t-\varepsilon x \mathrm{~d} \beta, \quad \mathrm{d} y=\mathscr{B}_{1}(x, y, t) \mathrm{d} t .
$$

where $\beta=\{\beta(t) ; t>0\}$ denotes standart Wiener process. Here $\varepsilon$ represents noise parameters. Here we assume that the death rate of prey could be noisy, thus $d=d+\varepsilon \dot{\beta}(t)$. The presence of noise terms turns the model (2.1) into a system of stochastic differential equations. The numerical solutions of the system (3.1) can be found using Euler Maruyama method [23].

\section{Extinction probability}

The notion of extinction is one of the key subjects in population dynamics. In biological terms the extinction occurs in a population if there is no individual that can reproduce or create a new generation in a long term [12]. In mathematical terms a population goes to extinction with probability one if $\lim _{t \rightarrow \infty} \mathscr{X}(t)=0$, where $\mathscr{X}$ is the density of a population. Thus the conditions for which the prey and predator species go to extinction can be found in a fluctuating environment as given in system (3.1).

In this context from the first bit of stochastic equation (3.1) it can be written that

$$
\mathrm{d} x=\left[r_{0} x f_{1}(k, y)-d x-a x^{2}-p x y\right] \mathrm{d} t-\varepsilon x \mathrm{~d} \beta .
$$


Considering $U(t)=\ln x(t)$ and $V(t)=\ln y(t)$ and applying Itô formula, the equation (4.1) can be written as

$$
\begin{aligned}
\mathrm{d} \ln x(t) & =\left[\frac{\partial U}{\partial t}+\mathscr{A}_{1}(x, y, t) \frac{\partial U}{\partial x}+\frac{1}{2}(-\varepsilon x)^{2} \frac{\partial^{2} u}{\partial x^{2}}\right] \mathrm{d} t+(-\varepsilon x) \frac{\partial U}{\partial x} \mathrm{~d} \beta \\
& =\left(\mathscr{A}_{2}(x, y, t)-\frac{\varepsilon^{2}}{2}\right) \mathrm{d} t-\varepsilon \mathrm{d} \beta
\end{aligned}
$$

with initial values $U(0)=\ln U_{0}$ and $V(0)=\ln V_{0}$. Equation (4.3) can be rewritten as

$$
\begin{aligned}
\mathrm{d} U(t) & =\left(\mathscr{A}_{2}\left(\mathrm{e}^{U}, \mathrm{e}^{V}, t\right)-\frac{\varepsilon^{2}}{2}\right)-\varepsilon \mathrm{d} \beta \\
& =\left(\frac{r_{0}}{1+k \mathrm{e}^{V}}-d-a \mathrm{e}^{U}-p \mathrm{e}^{V}-\frac{\varepsilon^{2}}{2}\right) \mathrm{d} t-\varepsilon \mathrm{d} \beta \\
& \leq\left(r_{0}-d-a \mathrm{e}^{U}-\frac{\varepsilon^{2}}{2}\right) \mathrm{d} t-\varepsilon \mathrm{d} \beta \\
\mathrm{d} \ln x & \leq\left(r_{0}-d-a x-\frac{\varepsilon^{2}}{2}\right) \mathrm{d} t-\varepsilon \mathrm{d} \beta
\end{aligned}
$$

Taking $\tilde{f}(x)=r_{0}-d-a x-\varepsilon^{2} / 2$ and finding the supremum of $\tilde{f}$ one can derive that $\tilde{f}^{\prime}(x)=-a<0$. This implies that $\tilde{f}$ is an decreasing function and has maximum at $x=0$. Thus it can be written that $\tilde{f}(0)=r_{0}-d-\varepsilon^{2} / 2$. From equation (4.4) it follows that

$$
\ln x \leq \ln x_{0}+\left(r_{0}-d-\frac{\varepsilon^{2}}{2}\right) t-\varepsilon \beta:=\mathscr{H}(t)
$$

from this

$$
\limsup _{t \rightarrow \infty} \frac{\ln x(t)}{t} \leq \limsup _{t \rightarrow \infty} \mathscr{H}(t)=r_{0}-d-\frac{\varepsilon^{2}}{2} .
$$

thus this concludes that one can have $\limsup _{t \rightarrow \infty} \frac{\ln x}{t} \leq 0$ with the condition $r_{0} \leq d+\frac{\varepsilon^{2}}{2}$ and this leads to $\lim _{t \rightarrow \infty} x(t)=0$.

\section{Numerical simulations}

In Figure 5.1, the evolution of prey density with respect to time is shown for different noise strengths where $\varepsilon=0$ (a), $\varepsilon=0.002$ (b), $\varepsilon=0.01$ (c) and $\varepsilon=0.02$ (d). The red and magenta lines represent the density of prey species deterministic and stochastic models respectively. The system is stable with damping oscillations, leading to a stable spiral with complex eigenvalues having negative real part, in the absence of noise as demonstrated in Fig. 5.1(a), corresponding to deterministic model. For a small perturbation, e.g. $\varepsilon=0.002$, the stochastic model demonstrates similar behaviour to deterministic model. Increasing noise term a bit further, irregular and large amplitude oscillatory dynamics for a stochastic model is observed, see 5.1(c,d). 


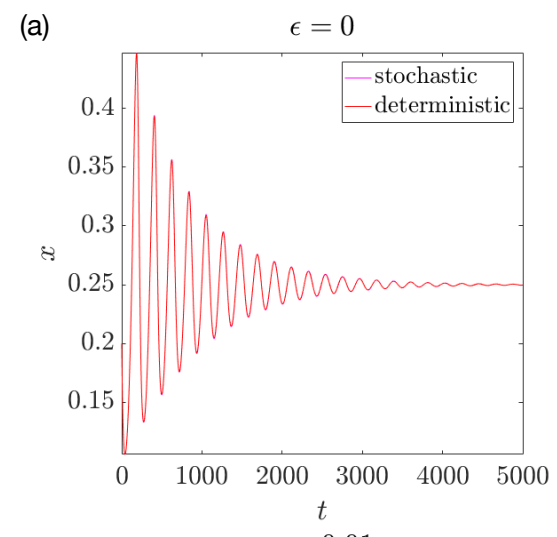

(b)

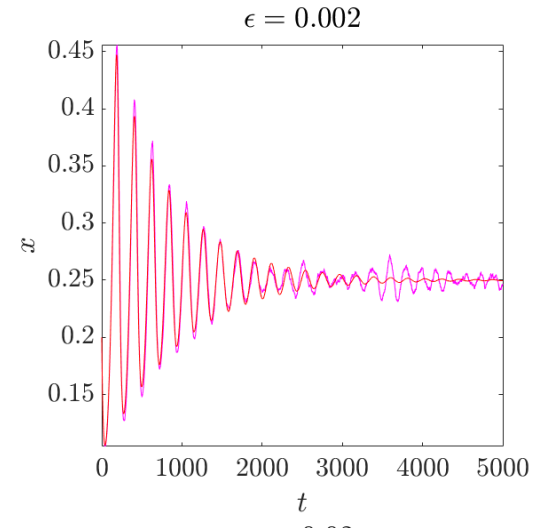

(c)

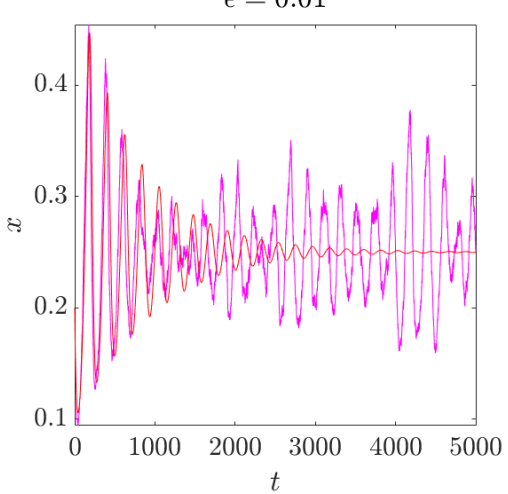

(d)

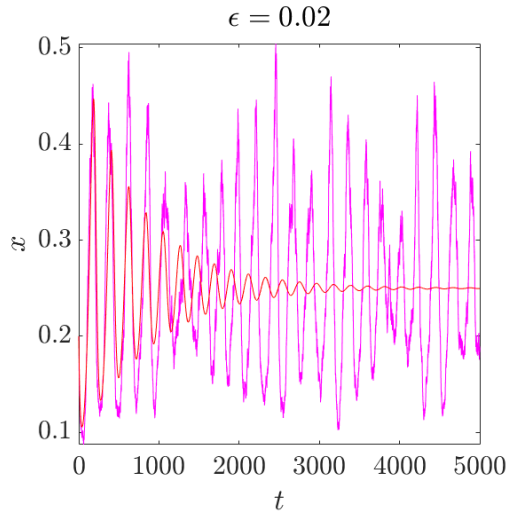

Figure 5.1: Comparison for time evolution of prey $(x)$ species between the deterministic model (2.1) and stochastic model (3.1) based on different values of noise parameter $\varepsilon=0(\mathrm{a}), \varepsilon=0.002(\mathrm{~b}), \varepsilon=0.01(\mathrm{c})$ and $\varepsilon=0.02(\mathrm{~d})$ with initial conditions $\left(x_{0}, y_{0}\right)=(0.2,0.1)$. Red and magenta colors respectively correspond to deterministic and stochastic dynamics of prey population.

(a)

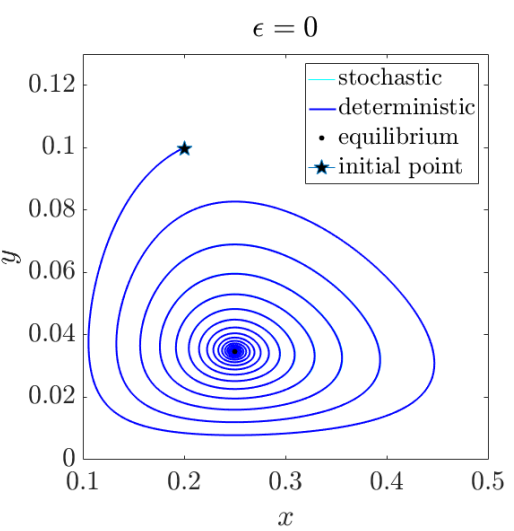

(c)

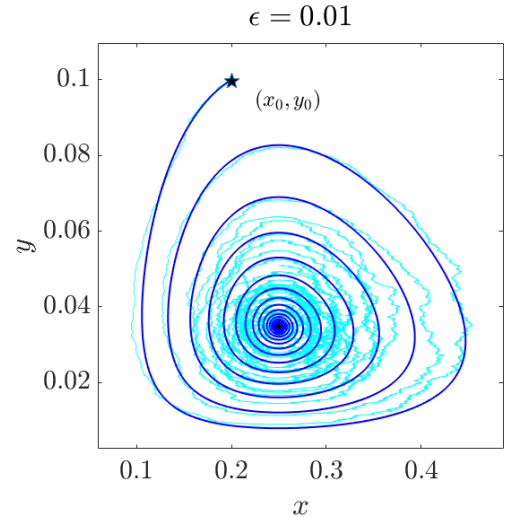

(b)

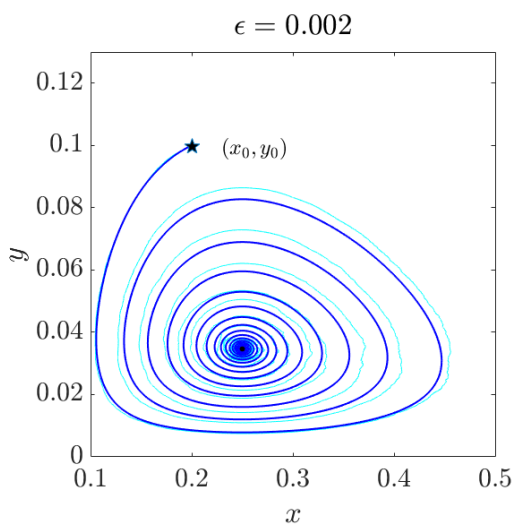

(d)

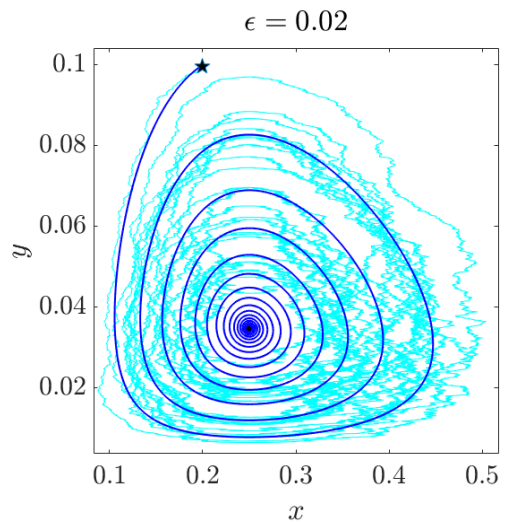

Figure 5.2: The comparison between phase portraits of two-component deterministic and stochastic prey-predator model, respectively presented in (2.1) and (3.1) for different values of noise terms $\varepsilon=0$ (a), $\varepsilon=0.002(\mathrm{~b}), \varepsilon=0.01(\mathrm{c})$ and $\varepsilon=0.02(\mathrm{~d})$ with initial conditions $\left(x_{0}, y_{0}\right)=(0.2,0.1)$. (black star). Steady state of the system is given by black point at $\left(x_{s}, y_{s}\right)=(0.25,0.0443)$. 
In Section 4 the analytical conditions for extinction of prey and predator species for the stochastic model are developed. Here it has been discussed that the extinction of species depends on the strength of noise term. It is found that high values of perturbation may lead extinction of both species. In Figure 5.3, it is demonstrated that prey and predator are wiped out from the system with the condition $r_{0} \leq d+\varepsilon^{2} / 2$ where $\varepsilon=0.2, r_{0}=0.03$ and $d=0.01$.

(a)

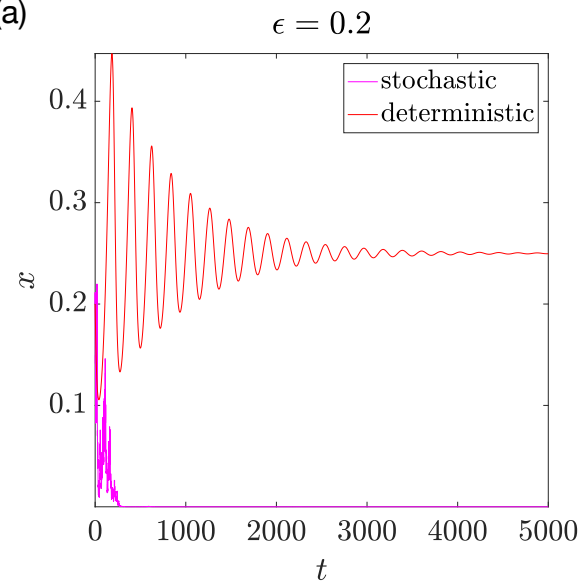

(b)

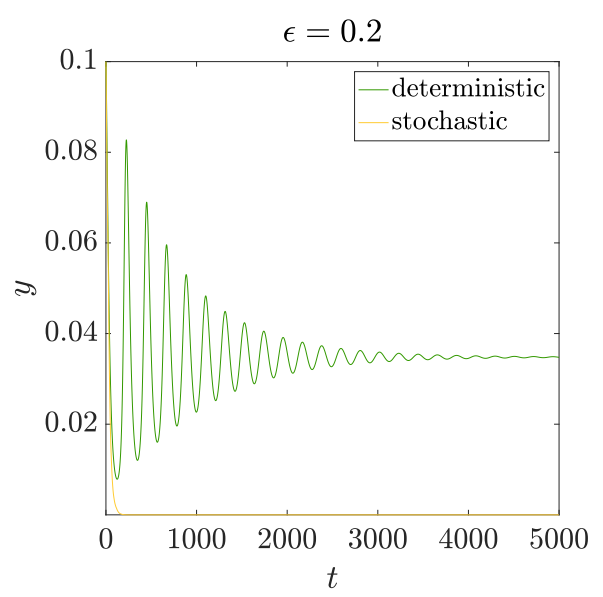

Figure 5.3: Extinction of prey and predator species with the condition $r_{0} \leq d+\varepsilon^{2} / 2$, where $\varepsilon=0.2, r_{0}=0.03$ and $d=0.01$.

In Figure 5.4, the corresponding phase portrait of the extinction case given in Fig. 5.3 is plotted for $\varepsilon=0.2$. The blue and cyan color respectively stand for deterministic and stochastic phase diagram. Here the initial condition is $\left(x_{0}, y_{0}\right)=(0.2,0.1)$. (black star) and the steady state of the system is given by black point at $\left(x_{s}, y_{s}\right)=(0.25,0.0443)$. Increasing the noise term, irregular nonperiodic random peaks are observed more frequently which can be also observed from the phase planes.

All simulations are performed using parameters: $r_{0}=0.03, k=0.1, d=0.01, a=0.01, p=0.5, m=0.05, c=0.4$ with increasing values of noise strengths $(\varepsilon)$.

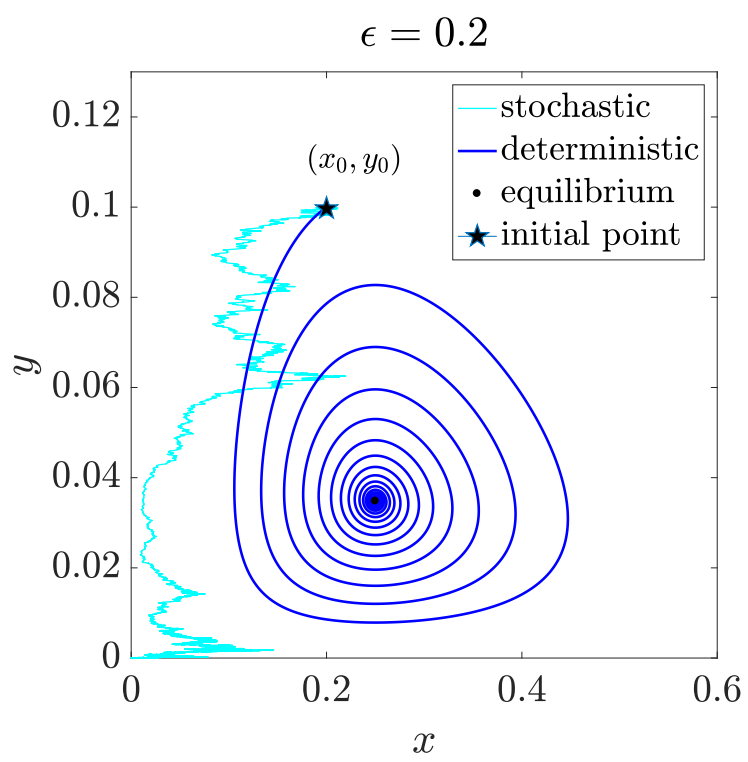

Figure 5.4: Phase diagram of the extinction state for prey and predator populations corresponding to Figure (5.3). 


\section{Conclusion}

Since natural fluctuations appear in many biological system, analyses performed on prey-predator type interactions with stochastic effects are much realistic compared to deterministic models. In this paper, the fear effect in a population model with a linear functional response is considered with random perturbation in prey's death rate. The model in the absence of white Gaussian noise is based on the paper written by Wang et al. [17], though without taking stochastic effects into account. Here a further analysis of the deterministic model including its positivity is presented. Compared to its deterministic version, it is also demonstrated that the high and low values of noise strength, denoted with $\varepsilon$, in the stochastic system give rise to rich spectrum of interesting results. Furthermore, excessive noise strength may induces both species to undergo extinction with $r_{0} \leq d+\varepsilon^{2} / 2$. This result is also biologically understandable as high environmental fluctuations may have a drastic impact on the populations and may lead extinction.

Numerical bifurcation is performed for different parameters and a transcritical point where stability changes is observed. In fact, linear functional response is the simplest choice and one could expect more interesting dynamics with other functional responses such as Holling type II and Holling type III. Although it is more challenging to perform analytical results for stochastic system, the theoretical conditions where prey and predator species undergo extinction are determined. Here it is found that high level of noise increase the probability of both species to be wiped out from the system.

One straightforward extension of this work would be to incorporate local and non-local delay terms in the model. In fact the interactions between prey and predator are not straightforward and require some time lag, e.g. gestation period. Then the stochastic model would be extended to comprise delay terms. It is well known that delay term in the model supports periodic oscillations, where Hopf bifurcation may occur through a limit cycle around the coexisting state. Therefore, more complex behaviour in the dynamics of both species are expected [24].

\section{Acknowledgements}

The author is thankful to the editor and the anonymous reviewers for their helpful comments and suggestions.

\section{Funding}

There is no funding for this work.

\section{Availability of data and materials}

Not applicable.

\section{Competing interests}

No potential conflict of interest was reported by the author.

\section{Author's contributions}

The author developed the mathematical formulas for stochastic model and performed corresponding numerical simulations, the author has also written the original manuscript and gave final approval of the current version and any revised version to be submitted to the journal.

\section{References}

[1] S. Mondal, G. P. Samanta, Dynamics of a delayed predator-prey interaction incorporating nonlinear prey refuge under the influence of fear effect and additional food, J. Phys. A. Math. Theor., 53(29) (2020), 295601.

[2] C.S. Holling, The components of predation as revealed by a study of small-mammal predation of the European Pine Sawfly1, Can. Entomol., 91(5) (1959), 293-320.

[3] C.S. Holling, Some characteristics of simple types of predation and parasitism1, Can. Entomol., 91(7) (1959), $385-398$.

[4] C.S. Holling, The functional response of predators to prey density and its role in mimicry and population regulation, Mem. Entomol. Soc. Can., 97(45) (1965), 5-60.

[5] S. Creel, D. Christianson, S. Liley, J. A. Winnie, Predation risk affects reproductive physiology and demography of elk, Science, 315(5814) (2007), 960-960.

[6] K. B. Altendorf, J. W. Laundré, C. A. López González, J. S. Brown, Assessing effects of predation risk on foraging behaviour of mule deer, J. Mammal., 82(2) (2001), 430-439.

[7] L.Y. Zanette, A. F. White, M. C. Allen, M. Clinchy, Perceived predation risk reduces the number of offspring songbirds produce per year, Science, 334(6061) (2011), 1398-1401.

[8] S. Pal, S. Majhi, S. Mandal, N. Pal, Role offear in a predator-prey model with Beddington-DeAngelis functional response, Z. Nat. Forsch. A., 74(7) (2019), 581-595.

[9] X. Wang, X. Zou, Modeling the fear effect in predator-prey interactions with adaptive avoidance of predators, Bull. Math. Biol., 79(6) (2017), 1325-1359.

[10] S.K. Sasmal, Y. Takeuchi, Dynamics of a predator-prey system with fear and group defense, J. Math. Anal. Appl., 481(1) (2020), 123471.

[11] S. Pal, N. Pal, S. Samanta, J. Chattopadhyay, Fear effect in prey and hunting cooperation among predators in a Leslie-Gower model, Math. Biosci. Eng., 16(5) (2019), 5146-5179. 
[12] A. Das, G. Samanta, A prey-predator model with refuge for prey and additional food for predator in a fluctuating environment, Physica A, 538 (2020), 122844 .

[13] A. Das, G. P. Samanta, Modeling the fear effect on a stochastic prey-predator system with additional food for the predator, J. Phys. A. Math. Theor.,51(46) (2018), 465601.

[14] C. Xu, G. Ren, Y. Yu, Extinction analysis of stochastic predator-prey system with stage structure and crowley-martin functional response, Entropy, 21(3) (2019), 252.

[15] Y. Cai, X. Mao, Stochastic prey-predator system with foraging arena scheme, Appl. Math. Model., 64 (2018), $357-371$.

[16] J. Roy, S. Alam, Fear factor in a prey-predator system in deterministic and stochastic environment, Physica A, 541 (2020), 123359.

[17] X. Wang, L. Zanette, X. Zou, Modelling the fear effect in predator-prey interactions, J. Math. Biol., 73(5) (2016), 1179-1204.

[18] L. van Veen, M. Hoti, Saddle-node-transcritical interactions in a stressed predator-prey-nutrient system, arXiv prep., 1809 (2018), 00108.

[19] A. Gökçe, Numerical bifurcation analysis for a prey-predator type interactions with a time lag and habitat complexity, Bitlis Eren Uni. Fen Bil. Derg., 10(1)(2020), 57-66.

[20] R. M. May, Stability and Complexity in Model Ecosystems, Princeton Uni. P., 2019.

[21] Q. Liu, L. Zu, D. Jiang, Dynamics of stochastic predator-prey models with Holling II functional response, Commun. Nonlinear. Sci. Numer. Simul., 37 (2016), 62-76.

[22] H. Qiu, M. Liu, K. Wang, Y. Wang, Dynamics of a stochastic predator-prey system with Beddington-DeAngelis functional response, Appl. Math. Comput., 219(4) (2012), 2303-2312.

[23] D. J. Higham, An algorithmic introduction to numerical simulation of stochastic differential equations, SIAM Rev. Soc. Ind. Appl. Math., 3(3) (2001),

[24] A. Gökçe, A mathematical study for chaotic dynamics of dissolved oxygen-phytoplankton interactions under environmental driving factors and time lag, Chaos Solitons Fractals, 151 (2021), 111268. 